\title{
Dissociation of the neural systems for working memory maintenance of verbal and nonspatial visual information
}

\author{
PIA RÄMÄ and JOSEPH B. SALA \\ Johns Hopkins University, Baltimore, Maryland \\ and \\ JOSEPH S. GILLEN, JAMES J. PEKAR, and SUSAN M. COURTNEY \\ Kennedy Krieger Institute, Baltimore, Maryland \\ and Johns Hopkins University, Baltimore, Maryland
}

\begin{abstract}
Working memory for names and faces was investigated to ascertain whether verbal and nonspatial visual information is maintained in working memory by separate neural systems. The subjects performed a delayed match-to-sample task for famous or unfamous faces and names and a sensorimotor control task. Several occipital, temporal, parietal, and prefrontal areas were activated during all memory delays, in comparison with the control delays. Greater delay activity for unfamous faces than for names was obtained in the right fusiform gyrus, right inferior frontal gyrus (IFG), right IFG/ precentral gyrus, and right medial superior frontal gyrus, whereas greater delay activity for unfamous names than for faces was observed in the precuneus, left insula/postcentral gyrus, and left IFG/ precentral gyrus. There was no significant difference in the prefrontal activity in the comparison between famous faces and names. Greater delay activity for famous names than for faces was obtained in visual association and parietal areas. The results indicate that there is a functional dissociation based on information type within the neural system that is responsible for working memory maintenance of verbal and nonspatial visual information.
\end{abstract}

The neural mechanisms underlying working memory for visuospatial (see, e.g., Carlson et al., 1998; Courtney, Petit, Maisog, Ungerleider, \& Haxby, 1998; Jonides et al., 1993; McCarthy et al., 1994), verbal (e.g., Awh et al., 1996; Braver et al., 1997; Cohen et al., 1997; Fiez et al., 1996; Jonides et al., 1997; Paulesu, Frith, \& Frackowiak, 1993) and nonspatial visual (e.g., Courtney, Ungerleider, Keil, \& Haxby, 1997; Haxby, Ungerleider, Horwitz, Rapoport, \& Grady, 1995; McCarthy et al., 1996) information have been studied extensively during recent years. It has been suggested that there is a domain-specific functional organization within the prefrontal cortex for working memory processing of spatial versus verbal and spatial versus nonspatial visual information (for reviews, see Courtney, Petit, Haxby, \& Ungerleider, 1998; Levy \& GoldmanRakic, 2000; Smith \& Jonides, 1999). Dorsal prefrontal regions, including the superior frontal sulcus, have been proposed to be specialized for working memory maintenance of visual locations (e.g., Courtney, Petit, Maisog,

P.R. is supported by the Academy of Finland. The authors thank Terri Brawner, Kila Morton, and the entire staff of the F. M. Kirby Research Center for Functional Brain Imaging, Kennedy Krieger Institute, where the data were acquired. We also thank Steve Yantis and Li Hsieh for comments on an earlier draft of the manuscript. Correspondence concerning this article should be addressed to S. M. Courtney, Department of Psychology, Room 132, Ames Hall, Johns Hopkins University, 3400 N. Charles Street, Baltimore, MD 21218 (e-mail: courtney@jhu.edu). et al., 1998; Courtney, Ungerleider, Keil, \& Haxby, 1996), whereas ventral prefrontal regions are more active for the maintenance of nonspatial visual and verbal information (e.g., Awh et al., 1996; Courtney, Petit, Maisog, et al., 1998; Courtney et al., 1997; Jonides et al., 1997; Nystrom et al., 2000).

Extensive evidence from behavioral, neuroimaging, and lesion studies has indicated that the neural systems for spatial and verbal working memory are distinct (for a review, see Baddeley, 1998; Smith \& Jonides, 1999). The right prefrontal cortex has been suggested to have a predominance for spatial working memory, and the left hemisphere for verbal working memory functions (e.g., Awh et al., 1996; Baker, Frith, Frackowiak, \& Dolan, 1996; Belger et al., 1998; Hanley, Young, \& Pearson, 1991; McCarthy et al., 1996; Nystrom et al., 2000; Paulesu et al., 1993; Smith, Jonides, \& Koeppe, 1996). The evidence for hemispheric laterality for nonspatial visual working memory has, however, been inconsistent. Some studies have shown a left-hemispheric predominance in ventral prefrontal regions for nonspatial visual working memory (Baker et al., 1996; Courtney, Petit, Maisog, et al., 1998; Smith et al., 1995), whereas in other studies, activation has been detected either bilaterally or only in the right prefrontal cortex (Courtney et al., 1996, 1997; Nystrom et al., 2000; Stern et al., 2000). In a positron emission tomography study of working memory for faces, in which the length of the delay between the sample and the test 
stimuli was parametrically varied, activation in the right prefrontal cortex tended to diminish at longer delays, whereas activation in the left prefrontal cortex increased (Haxby et al., 1995). It has been suggested that laterality effects in visual working memory may be influenced by the extent to which subjects engage in analytical or verbal, as opposed to image-based, rehearsal strategies during the performance of memory tasks (Courtney, Petit, Haxby, \& Ungerleider, 1998; Haxby et al., 1995).

There are, however, only a few imaging experiments that directly compare nonspatial visual and verbal working memory activation, and there is no conclusive evidence for segregation of these two systems (Nystrom et al., 2000; Paulesu et al., 1993; Salmon et al., 1996). Comparison of results across different imaging experiments also indicates that nonspatial visual and verbal working memory often generate similar patterns of activation in the prefrontal cortex (Fiez et al., 1996; Postle \& D’Esposito, 2000). These results may indicate that there is no segregation of nonspatial visual and verbal information in the prefrontal cortex. Alternatively, visual information might be recoded and maintained in working memory both visually and verbally, leading to coactivation of these two hypothesized systems.

In studies on long-term memory, there is evidence both for process- and material-specific involvement of frontal regions in the encoding and retrieval of verbal and visual information (e.g., McDermott, Buckner, Petersen, Kelley, \& Sanders, 1999; Tulving, Kapur, Craik, Moscovitch, \& Houle, 1994). Studies supporting material-specific processing in the frontal cortex have shown that separate neuronal systems are involved in memory encoding of verbal and nonspatial visual information (e.g., Brewer, Zhao, Desmond, Glover, \& Gabrieli, 1998; Iidaka, Sadato, Yamada, \& Yonekura, 2000; Kelley et al., 1998; Kirchhoff, Wagner, Maril, \& Stern, 2000; McDermott et al., 1999; Wagner et al., 1998). Encoding of words has been shown to activate the left frontal and temporal cortical regions, whereas the corresponding areas in the right hemisphere were activated during the encoding of pictures (Brewer et al., 1998; Iidaka et al., 2000; Kelley et al., 1998; Kirchhoff et al., 2000; Wagner et al., 1998). It has also been reported that the encoding of familiar visual objects that are namable and, thus, have access to both visual and verbal codes activates the extrastriate, temporal, and frontal areas bilaterally (Grady, McIntosh, Rajah, \& Craik, 1998; Kelley et al., 1998). There is also evidence suggesting process-specific involvement of the frontal cortex in the encoding and retrieval of information - that is, the left frontal cortex is recruited in encoding, and the right frontal cortex in the retrieval of information (Haxby et al., 1996; Tulving et al., 1994).

The aim of the present work was to ascertain whether verbal information and nonspatial visual information are maintained in working memory by separate neural systems. The subjects performed a delayed match-tosample (DMTS) task for famous names (FNs) or previously unfamiliar, unfamous names (UNs), famous faces
(FFs) or unfamous faces (UFs). We hypothesized that working memory for FNs and FFs that have access to both verbal and visual codes would activate common regions in the prefrontal cortex, whereas working memory for UNs and UFs would activate separate regions involved in verbal and nonspatial visual processing, respectively.

\section{METHOD}

\section{Subjects}

Fifteen right-handed, nonsmoking subjects ( 8 females) between the ages of 18 and 26 (mean, 21 years) participated in the study. The subjects were native English speakers and were screened for mental and physical health. They had no history of head injury or drug or alcohol abuse and no current use of medications that affect the central nervous system or cardiovascular function. The subjects gave written informed consent, and were paid $\$ 50$ for participating in the experiment. The experimental protocol was approved by the Review Board on the Use of Human Subjects of the Johns Hopkins University and by the Joint Committee on Clinical Investigations of the Johns Hopkins University School of Medicine. Before the scanning, the subjects completed a recognition test for the FFs and FNs used in the memory tasks. In the face recognition test, the subjects saw pictures of the FFs and UFs and were asked to indicate whether they had never seen the face before, had seen the face but were not able to recall the name, or were able to recall the proper name. In the name recognition test, the subjects read the names and were asked to indicate whether they had never heard the name, had heard the name but were not able to recall the face, or recognized the name and were able to recall the face. Only subjects who could recognize $80 \%$ or more of both the FFs and the FNs participated in the fMRI portion of the experiment.

\section{Stimuli}

Visual stimuli were presented with an LCD projector located outside of the scanning room, connected to a Power Macintosh G3 computer running SuperLab software. The stimuli were projected on a rear projection screen mounted inside the bore of the magnet, behind the subject's head. The subjects viewed the stimuli by using a mirror mounted at the top of the head coil. The stimuli were visually equivalent for all the memory conditions. The stimuli were grayscale images of FFs ( $n=24,12$ females) and previously unfamiliar UFs ( $n=24,12$ females). The FFs were of celebrities (actors, musicians, and politicians), and the UFs belonged to unknown individuals matched for age, sex, and facial expression with the FFs. A fixation cross and either an arbitrary complete name of an unknown person ( $n=24,12$ females) or the complete name of a famous person ( $n=24,12$ females) was superimposed on each of the images (Figure 1A). The subjects were instructed to pay attention to either the name or the face in the image and to maintain visual fixation at the cross throughout the trial. FNs were paired with UFs, rather than with FFs, in order to avoid possible interference effects. UNs were paired with FFs, rather than with UFs, so that all the tasks would be well matched to each other. Each name was superimposed on the images of two different faces. In the recognition tests completed before the scanning, the names were not superimposed on the faces. The numbers of syllables $[F(2,69)=0.95, p=.39]$ and letters $[F(2,69)=0.50, p=.61]$ of names were equal in each condition (4.2 syllables/ 12.2 letters for names corresponding to the FFs, 3.8/ 12.5 for FNs and 3.9/11.8 for UNs). Each face and name was presented six times during the experiment. Before the experiment, the subjects saw each face and name once during the recognition test and twice or three times during the task training. For the control (Ctrl) task, the experimental stimuli were phase scrambled in the Fourier domain, maintaining equal contrast, luminance, and 
A.

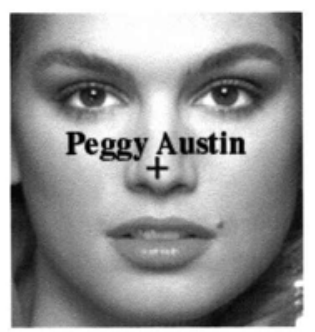

$\mathbf{F F}+\mathbf{U N}$

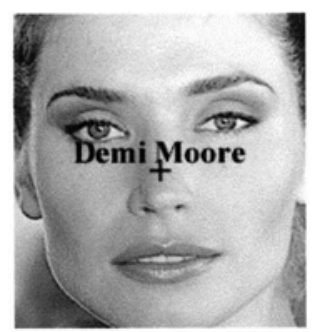

$\mathbf{U F}+\mathbf{F N}$

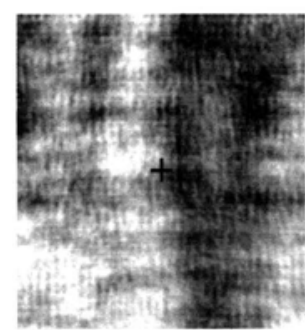

Phase-scrambled

B.

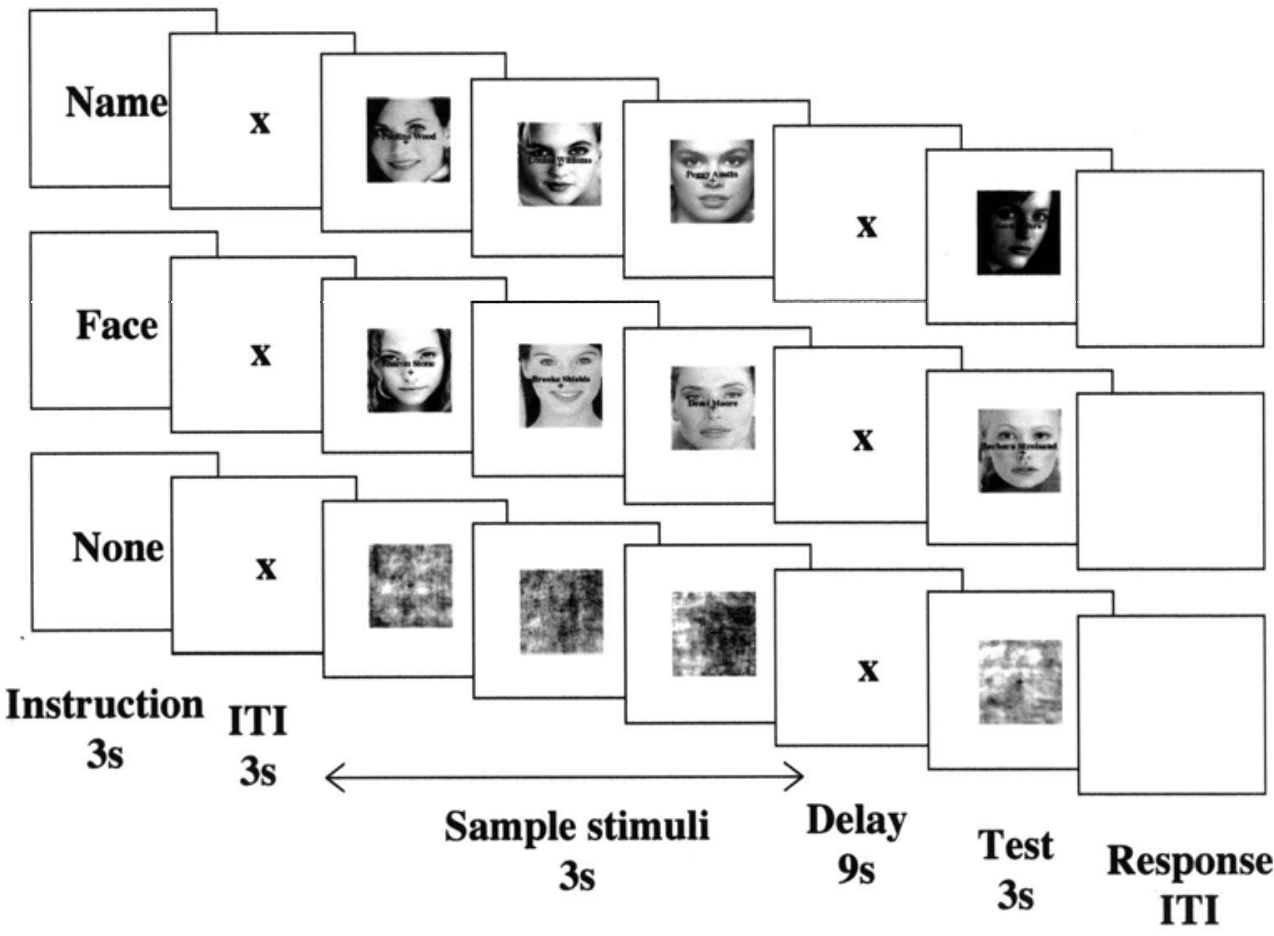

3s

Figure 1. A. Example of stimuli used for the tasks. Unfamous names (UN) were superimposed on the images of the famous faces (FF), and famous names (FN) on the images of the unfamous faces (UN). For the control tasks, the stimuli were phase scrambled in the Fourier domain. B. Illustration and timing of the delayed match-to-sample and control tasks.

frequency information of stimuli in both memory and Ctrl task conditions (Figure 1A).

\section{Working Memory and Sensorimotor Control Tasks}

There were four working memory tasks, in which the subjects were to remember FFs, FNs, UFs, or UNs. The subjects were presented with an instruction image (for $3 \mathrm{sec}$ ) consisting of the word face (for either the FF or the UF tasks), name (for either the FN or the UN tasks), or none (the Ctrl task) 6 sec before each trial, indicating which task was to be performed. In the DMTS tasks (Fig- ure 1B), a sample set of three stimuli was presented (one at a time, for $1 \mathrm{sec}$ each), which was followed by a memory delay of $9 \mathrm{sec}$, during which the subjects saw a blank screen with a fixation cross. Then, a test stimulus was presented for $3 \mathrm{sec}$, during which time the subject had to indicate, with a left or a right button press, whether or not the test stimulus was the same as any of those presented in the sample set. The face presented during the test period for a name task never matched one of the three faces presented during the sample phase. The name stimulus presented during the test period for a face task never matched one of the names presented during the sam- 
ple phase. Each subject was allowed to choose whether the right or the left hand would correspond to the match or the no-match response. One subject chose the left hand for match response, whereas the rest of the subjects chose the right hand. Following each trial, there was an intertrial interval of $9 \mathrm{sec}$. The subjects also performed a sensorimotor Ctrl task with no mnemonic demand, using scrambled stimuli. The scrambled stimuli were presented with the same timing as in the memory tasks, but the subjects were instructed that they need not remember the images but simply press both buttons when the test stimulus appeared.

During the scanning, eight runs were conducted. All four memory task conditions (FF, FN, UF, and UN) were presented in each run, in blocks of 4 trials for each task. Each block of 4 test trials was preceded and followed by 1 control trial. In each run, there were 16 memory test trials and 8 control trials. The order of tasks was counterbalanced across runs within each subject. The order of runs was counterbalanced across subjects. Altogether, there were 32 trials of each memory task condition and 16 of the Ctrl task. The reaction times and match/no-match responses were recorded during the scanning. After the scanning, each subject was asked to fill out a questionnaire rating the difficulty of each task, describing the mnemonic strategies used in his/her task performance, and stating whether he/she could distinguish or recognize any of the scrambled images.

\section{FMR Imaging and Data Analysis}

MR images were acquired with a 1.5 Tesla Philips Gyroscan ACS-NT MR scanner (Philips Medical Systems). A T1-weighted structural image (70 axial slices, $2.5 \mathrm{~mm}$, no gap, TR $=20 \mathrm{msec}$, $\mathrm{TE}=4.6 \mathrm{msec}$, flip angle $=30^{\circ}, \mathrm{FOV}=230 \mathrm{~mm}$, matrix $256 \times$ 256) was obtained before the functional scanning. During the performance of the tasks, the subjects underwent $\mathrm{T} 2 *$-weighted interleaved gradient-echo, echo-planar imaging (21 axial slices, $4.5 \mathrm{~mm}$ thickness, $0.5 \mathrm{~mm}$ gap, TR $=3,000 \mathrm{msec}$, TE $=40 \mathrm{msec}$, flip angle $=90^{\circ}, \mathrm{FOV}=230 \mathrm{~mm}$, matrix $64 \times 64$ ). The images were phase-shifted, using Fourier transformation to correct for slice acquisition time, then were motion-corrected, using automatic image registration (AIR) software (Woods, Grafton, Holmes, Cherry, \& Mazziotta, 1998), and were analyzed separately for each subject, using multiple regression (Friston et al., 1995; Haxby, Maisog, \& Courtney, in press) with NIH FIDAP software. Changes in neural activity were modeled as square-wave functions matching the time course of the events of the experimental tasks. The orthogonal squarewave contrasts were convolved with a gaussian model of the hemodynamic response, using previously experimentally derived average values for lag $(4.8 \mathrm{sec})$ and dispersion $(1.8 \mathrm{sec}$; Maisog, Clark, Courtney, \& Haxby, 1995). The convolved contrasts were the regressors of interest in the multiple regression analysis. Additional regressors were included to model sources of variance not related to the experimental manipulations (mean intensity between and linear drift within time series). Two separate multiple regression analyses were conducted, and analyses considered contributions from each of the main events (encoding, delay, and recognition) of the DMTS task separately. In the first analysis, the four memory task conditions were separately contrasted with their corresponding Ctrl tasks (e.g., FF vs. Ctrl task). The corresponding Ctrl task refers to the $\mathrm{Ctrl}$ trials preceding and following each block of four memory task trials. In the second analysis, FF and UF tasks were contrasted with FN and UN tasks, respectively, during the three main events of the task (encoding, delay, and recognition). The differences between the activations for the memory tasks, relative to their corresponding Ctrl tasks, were obtained through difference of differences direct comparisons (e.g., FF-Ctrl tasks vs. FN-Ctrl tasks). Furthermore, when orthogonal, the following four contrasts were included in the analysis to model additional sources of variance not related to the experimental manipulations of interest: visual stimulation versus no visual stimulation, visual objects versus scrambled objects, visual objects and scrambled objects versus instruction images, and working memory delays versus Ctrl delays. Each of these contrasts resulted in a $Z$ map for each subject.

$Z$ maps were registered into the Talairach coordinate system (Talairach \& Tournoux, 1988), resampled to $1 \mathrm{~mm}^{3}$, and spatially smoothed with a Gaussian kernel (2.5-mm FWHM). Average $Z$ maps were computed by dividing the sum of $Z$ values by the square root of the sample size, using AFNI software (Cox, 1996). In both average and single-subject $Z$ maps, an individual voxel $Z$ value of 2.33 was considered to be statistically significant, corresponding to $p<.01$. Statistical significance of a region of contiguous activated voxels was corrected for multiple comparisons according to the spatial extent, using a cluster size obtained from Monte Carlo simulation (Ward, 2000). In the Monte Carlo simulation, the threshold cluster size was estimated by using the number of voxels in the volume of each individual brain (excluding scull and the matrix outside the brain) and individual voxel probability of $p<.01$. The number of voxels in the individual masks of the brain varied between 16,282 and 24,297 (mean, 19,821). A given region was considered significantly activated at an experiment-wise $p<.05$ when it contained 7 or more (spatially smoothed averaged $Z$ maps) or 6 or more (spatially unsmoothed individual $Z$ maps) contiguous suprathreshold $3.59 \times$ $3.59 \times 5 \mathrm{~mm}$ voxels corresponding to cluster sizes of 451 and 387 $\mathrm{mm}^{3}$, respectively. Activations were anatomically localized in the individual (direct comparisons between delay activity for memory tasks) and/or averaged (direct comparisons between the tasks, and task relative to Ctrl task comparisons) maps using both EPI and T1weighted images.

\section{Region of Interest Analysis}

Regions of interest (ROIs) encompassing the inferior frontal gyrus/insula (IFG/insula), the IFG, the middle frontal gyrus (MFG), and the precentral gyrus (PreCG) were drawn in both hemispheres of a Talairach-transfor med brain according to Brodmann areas (BAs) and anatomical landmarks of the Talairach (Talairach \& Tournoux, 1988) and Damasio (Damasio, 1995) brain atlases. The IFG/ insula ROI included BAs 45 and 47 of the IFG $(z=-5.0$ to $10.0 \mathrm{~mm}$ ). The more superior IFG ROI included BAs 44 and 45 of the IFG $(z=16.0-30.0 \mathrm{~mm})$. The posterior border of the IFG ROI was the anterior bank of the precentral sulcus (PreCS) and the anterior border was the posterior bank of the inferior frontal sulcus (IFS). The MFG ROI included BAs 46, 10, and 9 of the MFG $(z=$ $5.0-30.0 \mathrm{~mm}$ ). The posterior border of the MFG ROI was the anterior bank of the IFS, and the anterior border was the posterior bank of the superior frontal sulcus (SFS). The PreCG ROI included BA 6 of the PreCG $(z=20.00-50.00 \mathrm{~mm})$. The posterior border of the PreCG was the anterior bank of the central sulcus, and the anterior border was the posterior bank of the PreCS.

The number of voxels significantly activated in each ROI for the comparison between delay periods of UF and UN tasks, relative to corresponding $\mathrm{Ctrl}$ tasks, was computed for each subject. The number of activated voxels was normalized by dividing by the total number of voxels in each ROI. An analysis of variance for repeated measures (BMDP2v; BMDP Statistical Software, Release 7.1) was used to test the main effects and interactions of task, hemisphere, and brain region on the number of suprathreshold voxels. A pairwise $t$ test was used to test the effect of task and hemisphere on the number of activated voxels in each ROI.

\section{RESULTS}

\section{Behavioral Data}

In the recognition test conducted before the scanning, the subjects were able to recognize and name, on average, $95 \%(S D, 5.09)$ of the FFs and to recall 99\% (SD, 1.47) of faces corresponding to the FNs. The subjects reported that they had seen, on average, $21 \%$ of the UFs before. The UFs 
were, however, of Scandinavian models previously unfamiliar to the group of subjects who participated in the study. It is possible that because of some similarity of the facial features of models and celebrities, our subjects felt familiar with some of the UFs. However, the subjects reported that they were not able to name these unknown individuals, and thus, this familiarity effect should not have interfered with our results. Behavioral data (the number of correct responses and reaction times during scanning) were obtained from 10 subjects, and a questionnaire regarding performance strategies was obtained from 14 subjects. The subjects were very accurate in all the task conditions. There was, however, a significant effect of task for the number of correct responses $[F(3,26)=7.81$, $p<.0005]$. The subjects were significantly more accurate in the task for UFs $(96 \%)$ than in that for UNs $(90 \%)$, whereas there was no significant difference between the tasks for FFs $(97 \%)$ and FNs $(98 \%)$. There was no significant effect of task on the reaction times $[F(3,6)=$ $0.62, p=.61]$. The reaction times were $1,153,1,200$, 1,065 , and $1,133 \mathrm{msec}$ for UFs, UNs, FFs, and FNs, respectively. Despite the better accuracy on UFs than on UNs, however, most subjects reported the UF task to be subjectively more difficult than the UN task. In the task for FFs, most of the subjects reported having used a verbal strategy during their task performance $(n=12), 1$ subject used a visual strategy, and 1 a combination of verbal and visual strategies. Most of the subjects $(n=11)$ reported that they made verbal descriptions of UFs in order to be able to memorize them, and the rest of the subjects visualized the faces. In the tasks for FNs and UNs, all the subjects reported having used a verbal strategy.

\section{f MRI Data}

Areas activated during the delay phase of the memory tasks relative to the control tasks (Tables 1 and 2). Several extrastriate and prefrontal areas showed greater activity during working memory delays than during Ctrl delays (Figures 2A-2D). Here, we describe the results from the averaged $Z$ maps.

In the occipitotemporalcortex, the fusiform gyrus (FG) was bilaterally activated during all the tasks. The lingual gyrus (LG) was activated for FFs and FNs. The cuneus (Cun) was bilaterally activated for both face tasks. The left middle temporal gyrus (MTG) was activated for both name tasks. The left superior temporal gyrus (STG) was activated only for UFs. The right lateral occipital gyrus (LOG) was activated only for FNs.

Table 1

Delay Activity for Unfamous Faces and Names Versus Control

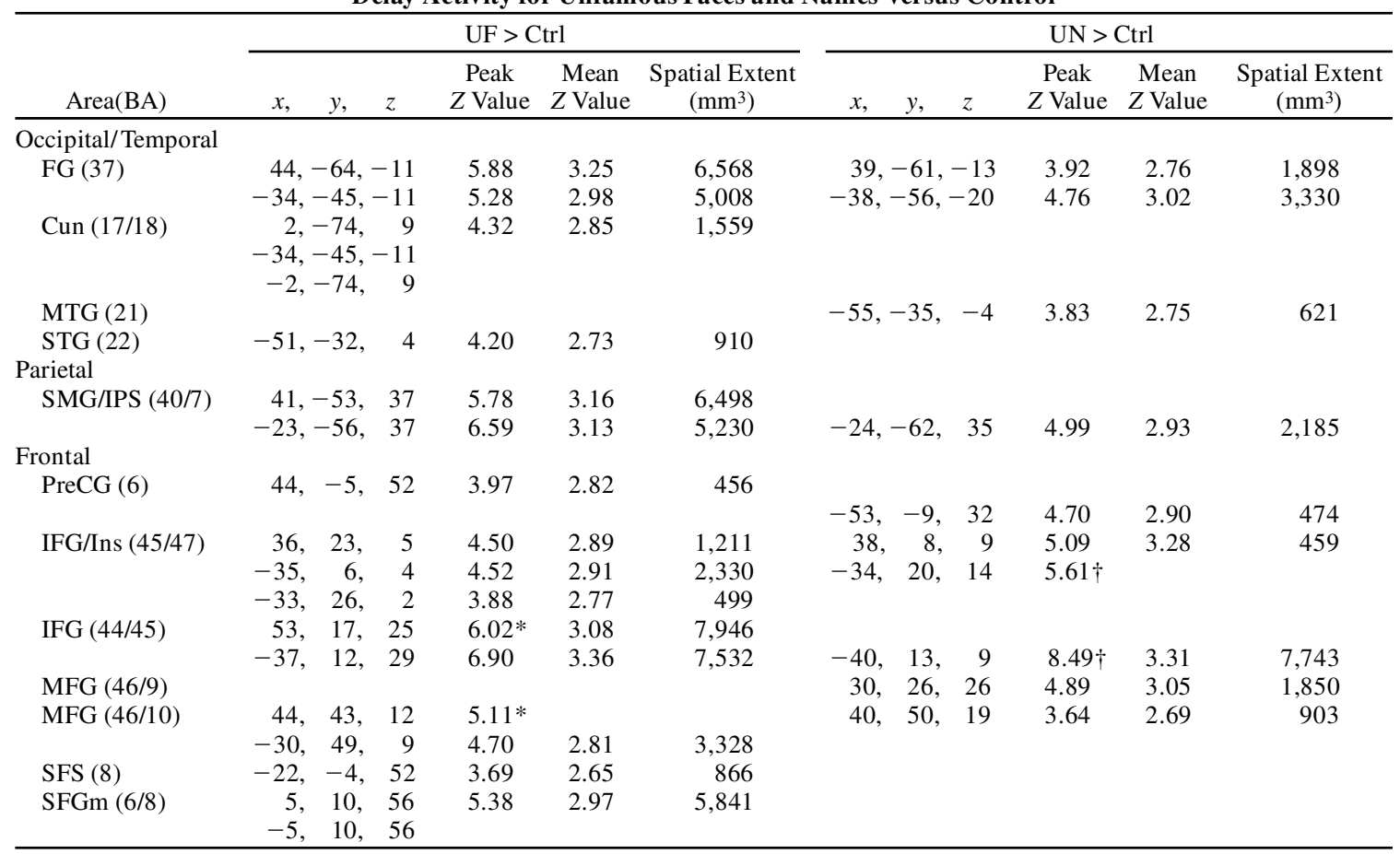

Note-Areas of significant activity, the peak and mean $Z$ values, the spatial extent of a given activity, and the Talairach coordinates of the maximum $Z$ value within each region during the delay period of the memory tasks relative to control (Crtl) tasks. UF, unfamous face; UN, unfamous name; FG, fusiform gyrus; Cun, cuneus; MTG, middle temporal gyrus; STG, superior temporal gyrus; SMG/IPS, supramarginal gyrus/intraparietal sulcus; PreCG, precentral gyrus; IFG/Ins, inferior frontal gyrus/insula; IFG, inferior frontal gyrus; MFG, middle frontal gyrus; SFS, superior frontal sulcus; SFGm, medial wall of the superior frontal gyrus. *The cluster included two separate activation loci with local maxima in both the IFG and the MFG. ${ }^{\dagger}$ The cluster included two separate activation loci with local maxima in both the IFG and the IFG/Ins. 
Table 2

Delay Activity for Famous Faces and Names Versus Control

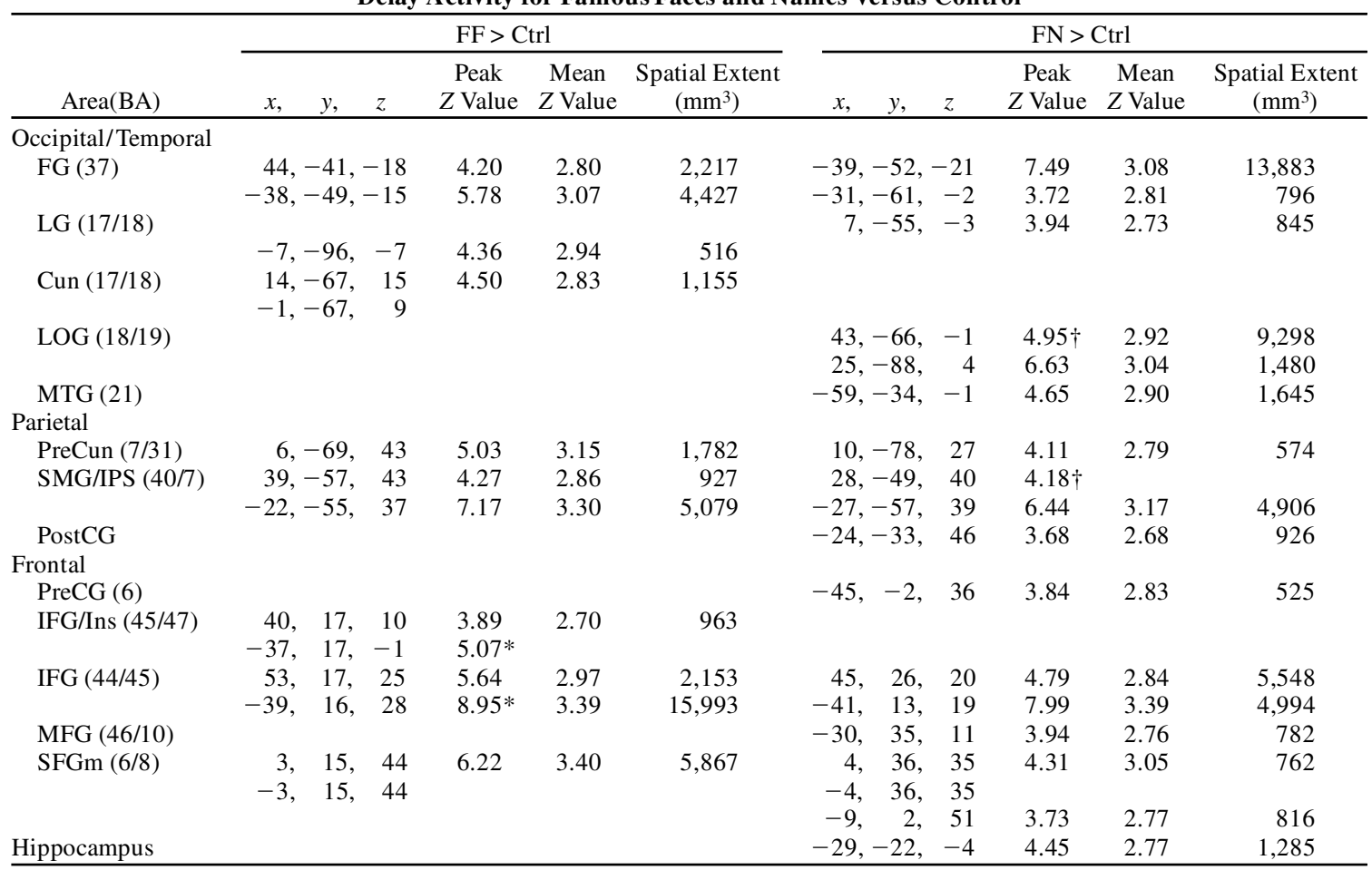

Note-Areas of significant activity, the peak and mean $Z$ values, the spatial extent of a given activity, and the Talairach coordinates of the maximum $Z$ value within each region during the delay period of the memory tasks relative to control (Ctrl) tasks. FF, famous face; FN, famous name; FG, fusiform gyrus; LG, lingual gyrus; LOG, lateral occipital gyrus; Cun, cuneus; MTG, middle temporal gyrus; PreCun, precuneus; SMG/IPS, supramarginal gyrus/intraparietal sulcus; PostCG, postcentral gyrus; PreCG, precentral gyrus; IFG/Ins, inferior frontal gyrus/insula; IFG, inferior frontal gyrus; MFG, middle frontal gyrus; SFGm, medial wall of the superior frontal gyrus. *The cluster included two separate activation loci with local maxima in both the IFG and IFG/Ins. †The cluster included two separate activation loci with local maxima in both the LOG and the SMG/IPS.

In the parietal cortex, all the tasks activated the left supramarginal gyrus and the intraparietal sulcus (SMG/ IPS). All but the UN task also activated the right SMG/ IPS. The right precuneus (PreCun) was activated during the FF and FN tasks.

In the frontal cortex, the IFG corresponding to BAs 44 and 45 was consistently activated during all the task conditions. Activation was detected in the left hemisphere during the UN task and bilaterally during the other tasks. The IFG/Insula (BAs 45/47) was bilaterally activated during all but the FN task. The MFG (BAs 46/10) was activated during the UF (bilateral), UN (right), and FN (left) tasks. The medial wall of the superior frontal gyrus (SFGm, BAs 6/8) was activated during all but the UN task, and the left SFS (BA 8) only during the UF task. The left PreCG (BA 6) was activated for both name tasks, and the right PreCG for only the UF task. The hippocampus was activated only for FNs.

Areas activated during the memory task for famous and unfamous faces relative to famous and unfamous names (Table 3). In the direct comparison of UF versus UN delays, differences in activation were observed in the occipitotemporal and prefrontal cortices. In averaged data, delay activity that was greater for UFs than for UNs was detected in the right FG, the right SFGm, the right IFG corresponding to BAs 44 and 45 , and the right IFG/ PreCG corresponding to BAs 44 and 6 (Figure 3A). Delay activity that was greater for UNs than for UFs was observed in the PreCun, the left IFG/PreCG (BAs 44/6), and the left insula/postcentral gyrus (insula/PostCG; Figure 3B). In the direct comparison between UFs and UNs during the encoding and recognition phases of the memory tasks, there were no significant differences in the amount of activity in either phase of the memory task.

In contrast with the comparisons between UFs and UNs, there was no difference in activation of prefrontal areas between the tasks for FFs versus FNs. Greater delay activity for FNs than for FFs was observed in several visual association and parietal areas (Table 3). No areas showed greater delay activity for FFs than for FNs. The PreCun (Talairach coordinates $-1,-46$, and 46 ; mean $Z$ value $=$ 2.64; peak $Z$ value $=3.48$; spatial extent, $488 \mathrm{~mm}^{3}$ ) was activated more for encoding of FNs than for that of FFs. 


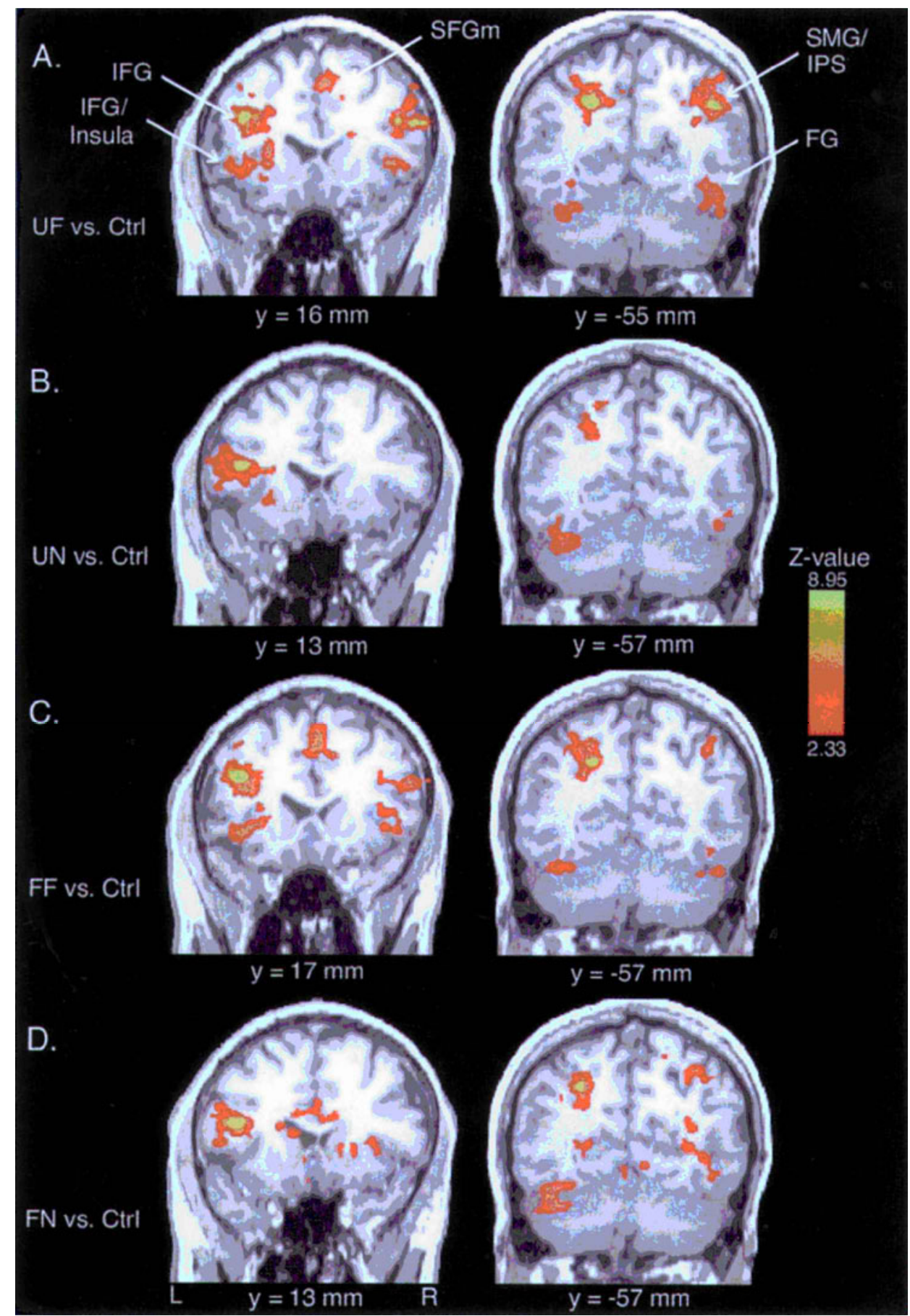

Figure 2. Coronal slices illustrating activity in group data during the delay period of the (A) unfamous face (UF), (B) unfamous name (UN), (C) famous face (FF), and (D) famous name (FN) tasks relative to their corresponding control (Ctrl) tasks in the prefrontal (left column) and extrastriatal (right column) regions.

No differences between FFs and FNs were observed during the recognition phase of the task.

The right IFG and right IFG/PreCG that were activated more for faces than for names and the left IFG/PreCG that was activated more for names than for faces during the delay period of the tasks were also activated more for the individual memory tasks, relative to their corresponding Ctrl tasks. The left insula/PostCG that was activated more for names than for faces was not more active for names, relative to the corresponding Ctrl task. Instead, there was a deactivation for faces relative to the corresponding Ctrl task in this region (Talairach coordinates 
Table 3

Direct Comparisons Between Working Memory Tasks

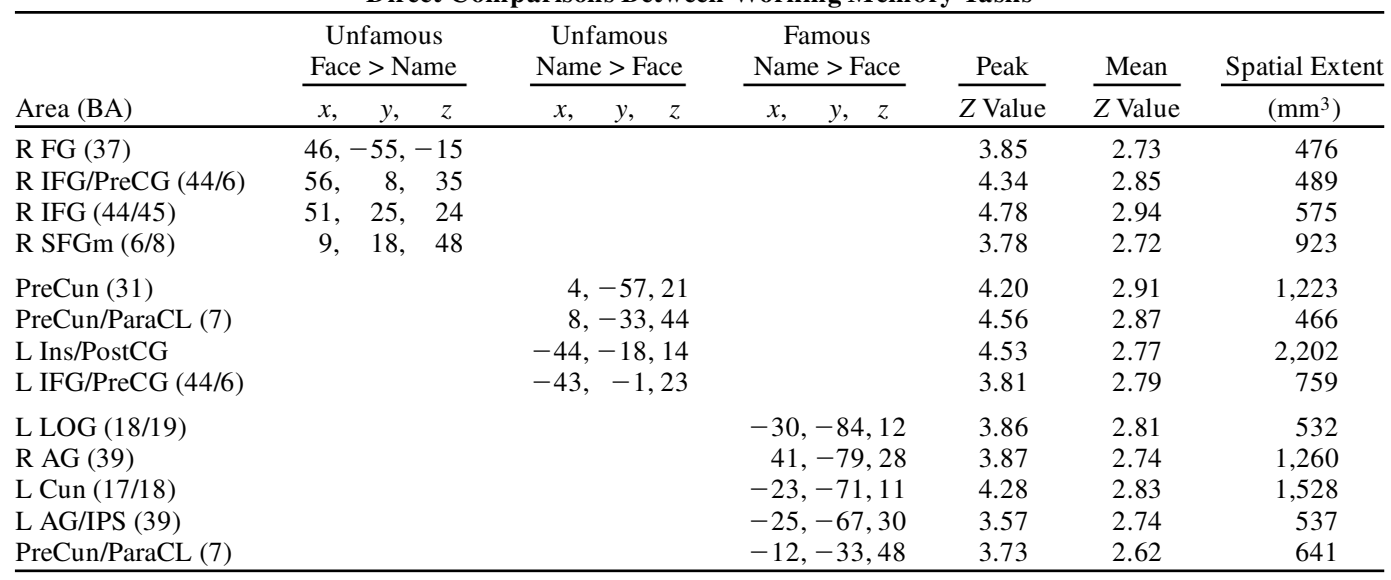

Note-Areas of significant activity, the peak and mean $Z$ values, the spatial extent of a given activity, and the Talairach coordinates of the maximum $\mathrm{Z}$ value within each region during the delay period of the memory tasks for faces versus names. R, right; L, left; FG, fusiform gyrus; LOG, lateral occipital gyrus; Cun, cuneus; AG, angular gyrus; IPS, intraparietal sulcus; PreCun, precuneus; ParaCL, paracentral lobulus; Ins/PostCG, insula/postcentral gyrus; IFG, inferior frontal gyrus; SFGm, medial wall of the superior frontal gyrus; PreCG, precentral gyrus.

$-56,-23$, and 20; mean $Z$ value $=2.99$; peak $Z$ value $=6.06 ;$ spatial extent, $6,052 \mathrm{~mm}^{3}$ ).

Individual subject and ROI analyses in frontal areas. In individual subjects, when corrected for multiple comparisons, the right IFG (BA 44/45) was activated more in 4 subjects, the bilateral IFG in 3 subjects, and the left IFG in 3 subjects for UFs than for UNs. The right IFG/PreCG (BA 44/6) was activated more in 3 subjects and the bilateral IFG/PreCG in 1 subject for UFs than for UNs. The left IFG/PreCG was activated more in 5 subjects and the bilateral IFG/PreCG in 3 subjects for UNs than for UFs. When corrected for multiple comparisons, 2 subjects showed a hemispheric double dissociation. The right IFG (45/45) was more active for faces than for names, and the left IFG/PreCG (44/6) more active for names than for faces. There was no correlation between the lateralization observed in individual subjects and the subjects' self-reported strategies.

The ROI analysis demonstrated that, across all ROIs, there was a tendency for an interaction between hemisphere and task in the number of suprathreshold voxels $[F(1,14)=3.45, p=.0845]$. The number of suprathreshold voxels for the UN task, relative to the corresponding Ctrl task, was significantly greater in the left than in the right hemisphere in the MFG $(p<.05)$ and PreCG $(p<$ $.05)$. In the IFG/insula, there was a tendency for a greater number of voxels in the left than in the right hemisphere for the $\mathrm{UN}$ task relative to the $\mathrm{Ctrl} \operatorname{task}(p=.098)$. No significant differences in the number of activated voxels were found between hemispheres for the UF task relative to the Ctrl task in any of the ROIs. Despite the significant differences in magnitude of activation within individual voxels demonstrated by the voxel-wise multiple regression analysis, when the number of activated voxels were compared between two memory tasks, there were no sig- nificant differences in any of the ROIs in the left hemisphere. In the right IFG/insula ( $p=.099)$ and the right IFG $(p=.069)$, there was a tendency for the number of activated voxels to be higher for the UF task than for UN task.

\section{DISCUSSION}

In the present work, we investigated whether verbal and nonspatial visual information is maintained in working memory by separate neural systems. We hypothesized that if separate neural systems subserve working memory for verbal and nonspatial visual information, UFs and UNs would activate separate regions, whereas working memory for FFs and FNs that are likely to be encoded both verbally and visually would activate common regions in the prefrontal cortex.

Greater delay activity for UFs than for UNs was detected in the right FG, the IFG, the IFG/PreCG, and the SFGm, whereas greater delay activity for UNs than for UFs was observed in the PreCun, the left insula/PostCG, and the left IFG/PreCG. It has been shown previously that the IFG is activated during both verbal and nonspatial visual working memory tasks (e.g., Belger et al., 1998; Courtney et al., 1996, 1997; Haxby et al., 1995; Jonides et al., 1997; McCarthy et al., 1996; Prabhakaran, Narayanan, Zhao, \& Gabrieli, 2000; Rypma, Prabhakaran, Desmond, Glover, \& Gabrieli, 1999; Smith et al., 1996). Verbal working memory has been shown to activate predominantly the left IFG, but the results concerning the lateralization of activation for nonspatial visual working memory have been inconclusive. The present results extend these previous findings by suggesting that there is a hemispheric double dissociation between verbal and nonspatial visual working memory processing. We found no differences in the pattern of activation for FFs versus 


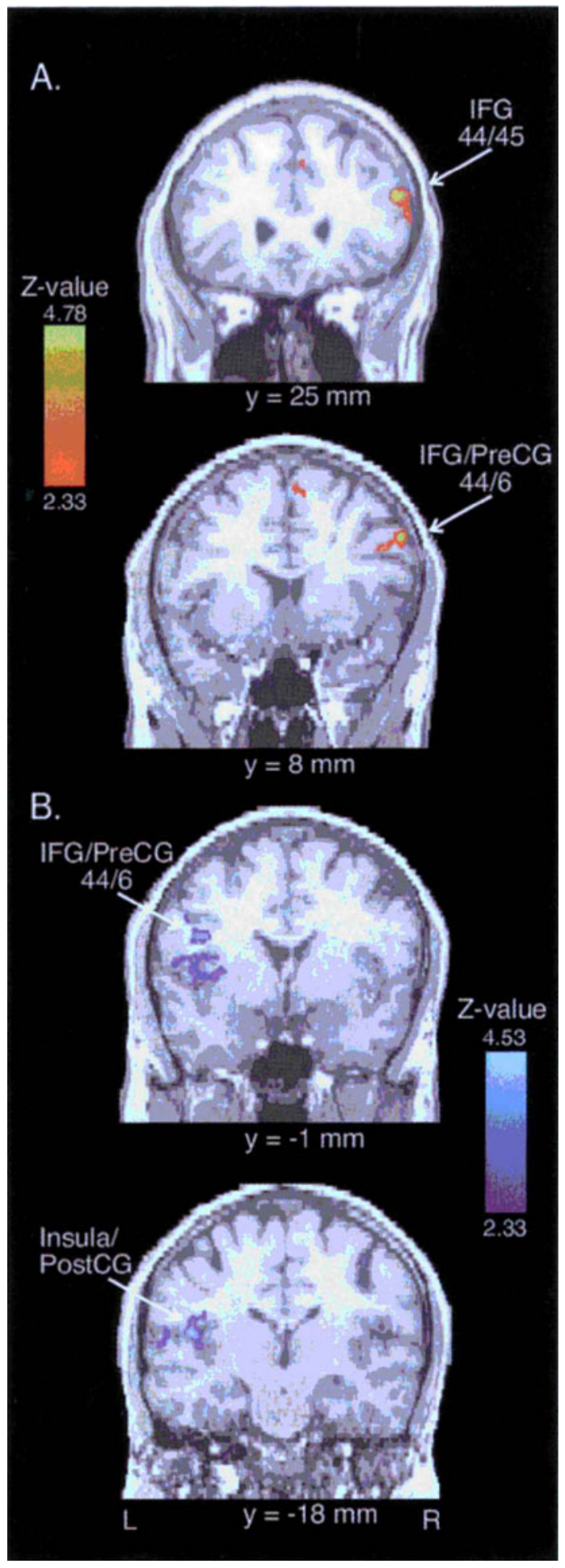

Figure 3. Grouped data from direct comparisons, corrected for multiple comparisons. (A) Delay activity greater for unfamous faces than for unfamous names in the right inferior frontal gyrus (IFG, BAs 44/45) and the right precentral gyrus (PreCG, BAs 44/6). (B) Delay activity greater for unfamous names than for unfamous faces in the left IFG/PreCG (BAs 44/6) and left insula postcentral gyrus (insula/ PostCG).
FNs in the prefrontal region, indicating that working memory for visual or verbal information that is associated with both verbal and visual codes activates common prefrontal neuronal networks.

The regions that showed a double dissociation between verbal and visual working memory did not exactly correspond anatomically between the left and the right hemispheres in the present study. Delay activity that was greater for faces than for names was observed in both the anteriorventral and the posterior portions of the IFG corresponding to BAs 44/45 and 44/6, respectively. For names, greater activation was detected only in the posterior portion of the IFG (IFG/PreCG, BAs 44/6). In previous studies on working memory processing of verbal information, activation related to rehearsal of phonological material has been located in the posterior portion of the left IFG corresponding to Broca's area (BA 44; Awh et al., 1996; Cohen et al., 1997; Jonides et al., 1997; Smith et al., 1996; for a review, see Smith \& Jonides, 1999). Activation associated with working memory maintenance of nonspatial visual information in previous studies has been located in more anterior and ventral portions of the IFG or in the anterior parts of the MFG (Courtney et al., 1996, 1997; for a review, see Smith \& Jonides, 1999).

Previously, it has been shown that the length of the delay influences the laterality of activity during nonspatial visual working memory tasks. Short (1-6 sec) delay periods cause more right-hemispheric activation, whereas longer delay periods cause bilateral or more left-hemispheric activation (Courtney, Petit, Haxby, \& Ungerleider, 1998; Haxby et al., 1995). The length of the delay may influence the subject's strategy. In the present study, many of our subjects reported having used a verbal strategy during the performance of the UF task, and the IFG was bilaterally activated in the comparison between the UF task and the Ctrl task. In the comparison between the UN task and the Ctrl task, activation in the IFG and the PreCG was detected only in the left hemisphere. It is possible that if we had used a shorter delay interval, our subjects would have engaged more in image-based than in analytical or verbal rehearsal strategies during the face task and, therefore, activated more exclusively the right hemisphere. In line with previous studies on working memory for nonspatial visual and verbal information, these results would suggest that bilateral ventral prefrontal areas are recruited during working memory maintenance of verbalizable nonspatial visual images, whereas the left ventral prefrontal cortex is more selectively involved in working memory for verbal information.

All the memory tasks in the present study activated a largely overlapping set of prefrontal regions, relative to the Ctrl tasks. Our results indicate that the dissociation observed in the ventral prefrontal cortex is a difference in the magnitude of activation for UFs versus UNs, whereas there was no significant difference for FFs versus FNs. This result may indicate a distributed representation of both verbal and nonspatial visual information in both hemispheres, with only a relative difference in empha- 
sis. Alternatively, verbal and nonspatial visual information may indeed be maintained by separate neural systems involving the left and the right frontal cortices, respectively, but both printed words and pictures may contain both visualizable and verbalizable information, leading to coactivation of both systems. The degree of coactivation may be different for different kinds of stimuli (in this case, famous vs. unfamous).

A recent study by Nystrom et al. (2000), in which working memory for letters and abstract shapes was directly compared during the performance of $n$-back tasks, showed that the right MFG (BA 46) was activated more for shapes than for letters and the left premotor cortex/superior temporal cortex (BAs 6/22) was activated more for letters than for shapes. Activation in these regions did not, however, exhibit an interaction between information type and memory load, which led the authors to suggest that there is no evidence for a dissociation in the mnemonic processing of verbal and nonspatial visual material. If both shapes and letters contain both verbal and visual information, one would expect an increase in activation in regions specialized for verbal and visual working memory during either task and, therefore, no information type $X$ load interaction. Thus, the Nystrom et al. results do not disprove the hypothesis that separate neural systems subserve verbal and nonspatial visual working memory.

Although the accuracy of the performance was relatively high for both UFs (96\%) and UNs (90\%), there was a significant difference in accuracy between the tasks. The subjects, however, reported that the UF task was subjectively more difficult than the UN task. Previous studies on working memory have reported either increased (e.g., Braver et al., 1997; Cohen et al., 1997; Postle, Stern, Rosen, \& Corkin, 2000) or decreased (e.g., Menon, Anagnoson, Mathalon, Glover, \& Pfefferbaum, 2001; Stevens, Goldman-Rakic, Gore, Fulbright, \& Wexler, 1998) activation correlated with task performance and/or difficulty. Rypma et al. (1999) reported that the left ventral prefrontal cortex was activated for a verbal memory task independent of the number of items to be maintained during the delay period but that, when the difficulty of the task was increased, additional areas in the bilateral MFG and SFG were recruited. Activation in the anterior cingulate gyrus has also been associated with increased difficulty of a verbal working memory task (Barch et al., 1997). However, there are no reports of simultaneous increased left-hemisphere and decreased right-hemisphere activation with increased difficulty or decreased performance. Therefore, it is unlikely that the small difference in accuracy between the tasks in the present study could explain the observed hemispheric double dissociation in the prefrontal regions related to the maintenance of names versus faces.

Greater delay activity for UNs than for UFs was also observed in the left insula/PostCG. The insula has been implicated in language processing (for a review, see Ardila, Benson, \& Flynn, 1997). Electrical stimulation of the posterior insula produces transient anomia (Ojemann $\&$ Whitaker, 1978), and lesions in this region are associated with Wernicke's aphasia and auditory agnosia (see
Ardila et al., 1997; Flynn, Benson, \& Ardila, 1999; Habib et al., 1995). The left insula/PostCG in the present study was not, however, more active for names, relative to the corresponding $\mathrm{Ctrl}$ task. Instead, this region was deactivated for faces, relative to the corresponding Ctrl task, which is in line with previous findings demonstrating a decrease in activation in several regions in the auditory association, postcentral somatosensory, and parietal cortices during a working memory task for faces, relative to the Ctrl (Courtney et al., 1996, Haxby et al., 1994).

In summary, the present results show that the pattern of prefrontal activity is different between working memory for UFs and UNs, suggesting a hemispheric dissociation in the neural systems for verbal and nonspatial visual working memory. However, the results also indicate that these two systems are frequently coactivated during working memory maintenance for nonspatial visual information and that the dissociation is based on relative strengths of activation modulated by information type.

\section{REFERENCES}

Ardila, A., Benson, D. F., \& Flynn, F. G. (1997). Participation of the insula in language. Aphasiology, 11, 1159-1169.

Awh, E., Jonides, J., Smith, E. E., Schumacher, E. H., Koeppe, R. A., \& KaTZ, S. (1996). Dissociation of storage and rehearsal in verbal working memory: Evidence from positron emission tomography. Psychological Science, 7, 25-31.

BADDEley, A. (1998). Recent developments in working memory. Current Opinion in Neurobiology, 8, 234-238.

Baker, S. C., Frith, C. D., Frackowiak, R. S., \& Dolan, R. J. (1996). Active representation of shape and spatial location in man. Cerebral Cortex, 6, 612-619.

Barch, D. M., Braver, T. S., Nystrom, L. E., Forman, S. D., Noll, D. C., \& Cohen, J. D. (1997). Dissociating working memory from task difficulty in human prefrontal cortex. Neuropsychologia, 35, 1373-1380.

Belger, A., Puce, A., Krystal, J. H., Gore, J. C., Goldman-Rakic, P. S., \& McCARThy, G. (1998). Dissociation of mnemonic and perceptual processes during spatial and nonspatial working memory using f MRI. Human Brain Mapping, 6, 14-32.

Braver, T. S., Cohen, J. D., Ny strom, L. E., Jonides, J., Smith, E. E., \& Noll, D. C. (1997). A parametric study of prefrontal cortex involvement in human working memory. Neurolmage, 5, 49-62.

Brewer, J. B., Zhao, Z., Desmond, J. E., Glover, G. H., \& Gabrieli, J. D. (1998). Making memories: Brain activity that predicts how well visual experience will be remembered. Science, 281, 1185-1187.

Carlson, S., Martinkauppi, S., Rämä, P., Salli, E., Korvenoja, A., \& Aronen, H. J. (1998). Distribution of cortical activation during visuospatial $n$-back tasks as revealed by functional magnetic resonance imaging. Cerebal Cortex, 8, 743-752.

Cohen, J. D., Perlstein, W. M., Braver, T. S., Nystrom, L. E., Noll, D. C., Jonides, J., \& SMITH, E. E. (1997). Temporal dynamics of brain activation during a working memory task. Nature, 386, 604-607.

Courtney, S. M., Petit, L., Haxby, J. V., \& Ungerleider,L. G. (1998). The role of prefrontal cortex in working memory: Examining the contents of consciousness. Philosophical Transactions of the Royal Society of London: Series B, 353, 1819-1828.

Courtney, S. M., Petit, L., Maisog, J. M., Ungerleider, L. G., \& Hахву, J. V. (1998). An area specialized for spatial working memory in human frontal cortex. Science, 279, 1347-1351.

Courtney, S. M., Ungerleider, L. G., KeIl, K., \& HAXby, J. V. (1996). Object and spatial visual working memory activate separate neural systems in human cortex. Cerebal Cortex, 6, 39-49.

Courtney, S. M., Ungerleider,L. G., KeIl, K., \& Haxby, J. V. (1997). Transient and sustained activity in a distributed neural system for human working memory. Nature, 386, 608-611.

Cox, R. W. (1996). AFNI: Software for analysis and visualization of func- 
tional magnetic resonance neuroimages. Computers \& Biomedical Research, 29, 162-173.

DAMASIO, H. (1995). Human brain anatomy in computerized images. New York: Oxford University Press.

Fiez, J. A., Raife, E. A., Balota, D. A., Schwarz, J. P., Raichle, M. E., \& Petersen, S. E. (1996). A positron emission tomography study of the short-term maintenance of verbal information. Journal of Neuroscience, 16, 808-822.

Flynn, F. G., Benson, D. F., \& Ardila, A. (1999). Anatomy of the insula: Functional and clinical correlates. Aphasiology, 13, 55-78.

Friston, K. J., Holmes, A. P., Poline, J.-B., Grasby, P. J., Williams, C. R. \& Frackowiak, R. S. J. (1995). Analysis of fMRI time-series revisited. NeuroImage, 2, 45-53.

Grady, C. L., McIntosh, A. R., Rajah, M. N., \& Craik, F. I. (1998). Neural correlates of the episodic encoding of pictures and words. Proceedings of the National Academy of Sciences, 95, 2703-2708.

Habib, M., Daquin, G., Milandre, L., Royere, M. L., Rey, M., Lanteri, A., Salamon, G., \& Khalil, R. (1995). Mutism and auditory agnosia due to bilateral insular damage: Role of the insula in human communication. Neuropsychologia, 33, 327-339.

Hanley, J. R., Young, A. W., \& Pearson, N. A. (1991). Impairment of the visuo-spatial sketch pad. Quarterly Journal of Experimental Psychology, 43, 101-125.

Haxby, J. V., Horvitz, B., Ungerleider, L. G., Maisog, J. M., Pietrini, P., \& Grady, C. L. (1994). The functional organization of human extrastriate cortex: A PET-rCBF study of selective attention to faces and locations. Journal of Neuroscience, 14, 6336-6353.

Haxby, J. V., Maisog, J. M., \& Courtney, S. M. (in press). Multiple regression analysis of effects of interest in $\mathrm{fMRI}$ time series. In P. Fox, J. Lancaster, \& K. Friston (Eds.), Mapping and modelling the human brain. New York: Wiley.

Haxby, J. V., Ungerleider, L. G., Horwitz, B., Maisog, J. M., RAPOPORT, S. I., \& GRADY, C. L. (1996). Face encoding and recognition in the human brain. Proceeding sof the National Academy of Sciences, 93, 922-927.

Haxby, J. V., Ungerleider, L. G., Horwitz, B., Rapoport, S. I., \& GRADY, C. L. (1995). Hemispheric differences in neural systems for face working memory: A PET-rCBF study. Human Brain Mapping, 3, 68-82.

Iidaka, T., Sadato, N., Yamada, H., \& Yonekura, Y. (2000). Functional asymmetry of human prefrontal cortex in verbal and nonverbal episodic memory as revealed by fMRI. Cognitive Brain Research, 9, 73-83.

Jonides, J., Schumacher, E. H., Smith, E. E., Lauber, E. J., Awh, E., Minoshima, S., \& Koeppe, R. A. (1997). Verbal working memory load affects regional brain activation as measured by PET. Journal of Cognitive Neuroscience, 9, 462-475.

Jonides, J., Smith, E. E., Koeppe, R. A., Awh, E., Minoshima, S., \& Mintun, M. A. (1993). Spatial working memory in humans as revealed by PET. Nature, 363, 623-625.

Kelley, W. M., Miezin, F. M., McDermott, K. B., Buckner, R. L., Raichle, M. E., Cohen, N. J., Ollinger, J. M., Akbudak, E., Conturo, T. E., Snyder, A. Z, \& Petersen, S. E. (1998). Hemispheric specialization in human dorsal frontal cortex and medial temporal lobe for verbal and nonverbal memory encoding. Neuron, 5, 927-936.

Kirchhoff, B. A., Wagner, A. D., Maril, A., \& Stern, C. E. (2000). Prefrontal-temporal circuitry for episodic encoding and subsequent memory. Journal of Neuroscience, 20, 6173-6180.

LEVy, R. \& GoldMAN-RAKIC, P. S. (2000). Segregation of working memory functions within the dorsolateral prefrontal cortex. Experimental Brain Research, 133, 23-32.

Maisog, J. M., Clark, V. P., Courtney, S. M., \& Haxby, J. V. (1995). Estimating the hemodynamic response and effective degrees of freedom in functional MRI time series. Human Brain Mapping, 1, 147.

McCarthy, G., Blamire, A. M., Puce, A., Nobre, A. C., Bloch, G., Hyder, F., Goldman-Rakic, P., \& Shulman, R. G. (1994). Functional magnetic resonance imaging of human prefrontal cortex activation during a spatial working memory task. Proceedings of the $\mathrm{Na}$ tional Academy of Sciences, 91, 8690-8694.

McCarthy, G., Puce, A., Constable, R. T., Krystal, J. H., Gore, J. C., \& Goldman-Rakic, P. S. (1996). Activation of human pre- frontal cortex during spatial and nonspatial working memory tasks measured by functional MRI. Cerebral Cortex, 6, 600-611.

McDermott, K. B., Buckner, R. L., Petersen, S. E., Kelley, W. M., \& SANDERS, A. L. (1999). Set- and code-specific activation in frontal cortex: An fMRI study of encoding and retrieval of faces and words. Journal of Cognitive Neuroscience, 11, 631-640.

Menon, V., Anagnoson, R. T., Mathalon, D. H., Glover, G. H., \& Pfefferbaum, A. (2001). Functional neuroanatomy of auditory working memory in schizophrenia: Relation to positive and negative symptoms. Neuroimage, 13, 433-446.

Nystrom, L. E., Braver, T. S., Sabb, F. W., Delgado, M. R., Noll, D. C., \& Cohen, J. D. (2000). Working memory for letters, shapes, and locations: fMRI evidence against stimulus-based regional organization in human prefrontal cortex. NeuroImage, 11, 424-446.

Ojemann, G. A., \& Whitaker, H. A. (1978). Language localization and variability. Brain \& Language, 6, 239-260.

Paulesu, E., Frith, C. D., \& Frackowiak, R. S. J. (1993). The neural correlates of the verbal component of working memory. Nature, $\mathbf{3 6 2}$, 342-345.

Postle, B. R., \& D'Esposito, M. (2000). Evaluating models of the topographical organization of working memory function in frontal cortex with event-related fMRI. Psychobiology, 28, 132-145.

Postle, B. R., Stern, C. E., Rosen, B. R. \& Corkin, S. (2000). An fMRI investigation of cortical contributions to spatial and nonspatial visual working memory. Neuroimage, 11, 409-423.

Prabhakaran, V., Narayanan, K., Zhao, Z., \& Gabrieli, J. D. E. (2000). Integration of diverse information in working memory within the frontal lobe. Nature Neuroscience, 3, 85-90.

Rypma, B., Prabhakaran, V., Desmond, J. E., Glover, G. H., \& GabrieLI, J. D. (1999). Load-dependent roles of frontal brain regions in the maintenance of working memory. NeuroImage, 9, 216-226.

Salmon, E., Van der Linden, M., Collette, F., Delfiore, G., MaQuet, P., Degueldre, C., LuXen, A., \& Franck, G. (1996). Regional brain activity during working memory tasks. Brain, 119, 1617-1625.

SMITH, E. E., \& JonidES, J. (1999). Storage and executive processes in the frontal lobes. Science, 283, 1657-1661.

Smith, E. E., Jonides, J., \& Koeppe, R. A. (1996). Dissociating verbal and spatial working memory using PET. Cerebral Cortex, 6, 11-20.

Smith, E. E., Jonides, J., Koepre, R. A., Awh, E., Schumacher, E. H., \& Minoshima, S. (1995). Spatial versus object working memory: PET investigations. Journal of Cognitive Neuroscience, 7, 337-356.

Stern, C. E., Owen, A. M., Tracey, I., Look, R. B., Rosen, B. R. \& Petrides, M. (2000). Activity in ventrolateral and mid-dorsolateral prefrontal cortex during nonspatial visual working memory processing: Evidence from functional magnetic resonance imaging. NeuroImage, 11, 392-399.

Stevens, A. A., Goldman-Rakic, P. S., Gore, J. C., Fulbright, R. K., \& WeXler, B. E. (1998). Cortical dysfunction in schizophrenia during auditory word and tone working memory demonstrated by functional magnetic resonance imaging. Archives in General Psychiatry, 55, 1097-1103.

TAlairach, J., \& Tournoux, P. (1988). Co-planar stereotaxic atlas of the human brain. New York: Thieme.

Tulving, E., Kapur, S., Craik, F. I., Moscovitch, M., \& Houle, S. (1994). Hemispheric encoding/retrieval asymmetry in episodic memory: Positron emission tomography findings. Proceedings of the $\mathrm{Na}$ tional Academy of Sciences, 91, 2016-2020.

Wagner, A. D., Schacter, D. L., Rotte, M., Koutstaal, W., Maril, A., Dale, A. M., Rosen, B. R., \& Buckner, R. L. (1998). Building memories: Remembering and forgetting of verbal experiences as predicted by brain activity. Science, 281, 1188-1191.

WARD, B. D. (2000). Simultaneous inference for fMRI data [On-line]. Available: http://afni.nimh.nih.gov/afni/docpdf/AlphaSim.pdf.

Woods, R. P., Grafton, S. T., Holmes, C. J., Cherry, S. R., \& MazZIOTTA, J. C. (1998). Automated image registration: I. General methods and intrasubject, intramodality validation. Journal of Computer Assisted Tomography, 22, 139-152.

(Manuscript received December 19, 2000; revision accepted for publication May 15, 2001.) 